\title{
Fighting against Infodemic during COVID-19 Vaccination
}

\author{
Hüsna Çevik
}

Çankaya District Health Directorate, Ankara, Turkey

Please cite this article as: Çevik H. Fighting against Infodemic during COVID-19 Vaccination. Anatol J Family Med 2021;4(3):281-282.

Address for correspondence: Dr. Hüsna Çevik. Çankaya District Health Directorate, Ankara, Turkey

Phone: +90 5547390727

E-mail: saricahusna@gmail.com

Received Date: 03.05.2021

Accepted Date: 25.08 .2021

Published online: 29.12.2021

(C) Copyright 2021 by Anatolian Journal of Family Medicine -

Available online at

www.anatoljfm.org

OPEN ACCESS

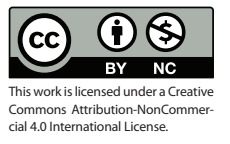

World Health Organization (WHO) mentioned the term "infodemic," which means a global epidemic of misinformation, on February 15, 2020, after a short while of the COVID-19 outbreak. WHO defines the infodemic as "too much information including false or misleading information in digital and physical environments during a disease outbreak." ${ }^{\prime[1]}$

Inactivated Sinovac-CoronaVac and Pfizer-BioNTech mRNA vaccines have been administered in Turkey so far. There are concerns about the long-term consequences of Pfizer-BioNTech since the vaccine has newer technology. People have many unscientific thoughts, such as the notion that mRNA vaccine will be injected into the human genome. Social media has been seen as the biggest source of the infodemic. ${ }^{[2,3]}$ This has caused some individuals to choose the inactivated virus vaccine if they have a choice. Despite this preference, I will talk about the growing infodemic related to the Sinovac-CoronaVac in this letter.

I am a family medicine specialist and working for a mobile vaccination team for elderly people and people who are bedridden in Ankara. Because of the convenience of storage and transportation, mobile vaccination teams use CoronaVac. While individuals vaccinated the first dose of the CoronaVac were more willing, some of them do not want to be vaccinated for the second dose due to the infodemic. People have a misconception that the second dose of the vaccine will make them sick, cause the real COVID-19 infection, and even cause death. When the source of this thought is questioned, it is seen that they have obtained this unreliable information through communication with others and social media. But there is no scientific evidence in the phase studies to prove these concerns. ${ }^{[4]}$

Sinovac-CoronaVac phase III clinical trials have announced efficacies in different countries from $50 \%$ to $91.25 \%$ and the effectiveness increases after the second dose. ${ }^{[5]}$ Although COVID-19 is seen as a life-threatening infection, it seems that the most important predictor of the desire for vaccination is trust in the vaccine. ${ }^{[6]}$ The antivaccine industry has used the COVID-19 pandemic to spread incorrect information about COVID-19 vaccines and make negative propaganda through social media platforms. When people search for COVID-19 related terms, an informing box appears to direct them to the websites of WHO, Centers for Disease Control and Prevention, or their country's Ministry of Health. In spite of this, the antivaccine industry affects nearly 60 -million people worldwide. ${ }^{[1,7]}$

One of the most important tasks of the health authorities to fight the pandemic is informing the public about the safety and efficacy of the COVID-19 vaccines and highlighting the detrimental effects of the illness, including death. This may positively affect vaccination rates. It should be emphasized that the primary care physicians, who have had the most important 
role since the beginning of the COVID-19 vaccination in our country, act with the awareness that patients have false assumptions about the second dose of the vaccination due to the infodemic. They should also inform the patients who come for their first dose appointments about the facts and myths related to the vaccines.

\section{REFERENCES}

1. Zarocostas J. How to fight an infodemic. Lancet 2020;395(10225):676.

2. Murphy J, Vallières F, Bentall RP, Shevlin M, McBride $O$, Hartman TK, et al. Psychological characteristics associated with COVID-19 vaccine hesitancy and resistance in Ireland and the United Kingdom. Nat Commun 2021;12(1):29.

3. Puri N, Coomes EA, Haghbayan H, Gunaratne K. Social media and vaccine hesitancy: new updates for the era of COVID-19 and globalized infectious diseases. Hum Vaccin Immunother 2020;16(11):2586-93.

4. Zhang Y, Zeng G, Pan H, Li C, Hu Y, Chu K, et al. Safety, tolerability, and immunogenicity of an inactivated SARS-CoV-2 vaccine in healthy adults aged $18-59$ years: a randomised, double-blind, placebo-controlled, phase 1/2 clinical trial. Lancet Infect Dis 2021;21(6):181-92.

5. Kim JH, Marks F, Clemens JD. Looking beyond COVID-19 vaccine phase 3 trials. Nat Med 2021;27:205-11.

6. Karlsson LC, Soveri A, Lewandowsky S, Karlsson L, Karlsson $\mathrm{H}$, Nolvi $\mathrm{S}$, et al. Fearing the disease or the vaccine: The case of COVID-19. Pers Individ Dif 2021;172:110590.

7. Burki T. The online anti-vaccine movement in the age of COVID-19. Lancet Digit Health 2020;2(10):e504-5. 